\title{
La section de glaciologie de la Société hydrotechnique de France : origine et évolution
}

\author{
Louis de Crécy
}

Louis REYNAud m'a demandé de vous faire un petit historique de la section de glaciologie de la SHF. D'autres, plus que moi, auraient été qualifiés pour cette tâche ! La section n'avait-elle pas 13 ans lorsqu'elle m'a accueilli ? Or, nous avons parmi nos membres actifs l'un de ceux qui participaient à la séance inaugurale de la « sous-section de glaciologie " de la SHF le 21 décembre 1948. j’ai nommé M. André PONCHET!

\section{Deux personnalités excep- tionnelles}

L'acte de naissance du 21 décembre 1948 était le résultat de la rencontre de deux personnages de stature exceptionnelle: Emile BARILLon et Jean MESSINES PAVIOT du SOURBIER. Le premier était Ingénieur Général du Génie maritime et Président du Comité technique de la SHF : esprit curieux de tout, il avait une connaissance approfondie de la plupart des montagnes de notre pays qu'il avait longuement parcourues à pied. En amateur éclairé, il avait une bonne connaissance des études existantes sur les glaciers de notre pays, depuis celles du Prince Roland BONAPARTE à la fin du siècle dernier jusqu'aux relevés systématiques des services des Eaux et Forêts.

L'Ingénieur Général des Eaux et Forêts Jean Messines Paviot du Sourbier, après une longue carrière savoyarde et de courts séjours aux postes de Nice et de Toulouse était responsable, depuis Grenoble où il habitait, de l'ensemble des services de Restauration des Terrains en Montagne en France. Il avait conduit les observations traditionnelles des forestiers sur les glaciers de Savoie et s'était particulièrement intéressé au problème des avalanches, suivant de près les premières études suisses au Weissfluhjoch dès avant la Seconde guerre mondiale. C'est à son instigation que plusieurs forestiers français alpins et pyrénéens, avaient, dès la fin de la guerre. été s'initier en Suisse aux problèmes des avalanches dont on pressentait la future importance.

\section{Les hydroélectriciens inves- tissent la montagne...}

A la même époque le pays souffrait d'une cruelle pénurie de ses ressources énergétiques et la toute jeune "Electricité de France» envoyait ses hydroélectriciens prospecter tous les sites équipables.

Avec Emosson, Argentières et d'autres projets d'altitude, les ingénieurs d'EDF étaient particulièrement intéressés par toutes données sur la fusion glaciaire alimentant leurs prises d'eau. Dans tous les autres sites de montagne, la connaissance des facteurs réglant le rythme de la fusion de la neige étaient encore plus essentiels pour leur prévision d'apport dans les retenues.

Pressés de "gagner de la chute ", les ingénieurs d'EDF se livraient donc à une prospection détaillée de la montagne qui n’allait pas sans inquiéter ses habitants, appelés, bien sûr, à bénéficier des routes modernes à construire, mais redoutant aussi les menaces d'expropriation et de submersion par les futurs barrages !

\section{3. ...et y rencontrent les fores- tiers!}

Dans leur investissement systématique de la montagne, les agents d'EDF ne pouvaient que rencontrer les forestiers, particulièrement ceux de la RTM, seuls services techniques de l'Etat vraiment présents dans les plus hautes vallées de montagne : traditionnellement, depuis près d'un siècle ils y géraient la forêt publique, assuraient la sécurité contre les torrents, employaient sur leurs chantiers le tropplein de main-d'œuvre locale, aidaient techniquement et subventionnaient la principale activité économique grâce aux crédits dits "d'améliorations pastorales " dont ils disposaient alors.

L'irruption des ingénieurs hydroélectriciens d'EDF dans le monde alors un peu figé de la montagne (l'explosion des sports d'hiver n'avait pas encore eu lieu !), ne pouvait que risquer de susciter des frottements avec les forestiers, voire des conflits !

\section{Neige et glace... un terrain neutre d'intérêt commun}

Le mérite de Monsieur MEssines fut d'avoir très vite trouvé la parade adéquate à un tel risque en cherchant à réunir sur le terrain neutre les « adversaires" potentiels. Il avait compris que leur formation commune d'ingénieurs et leur commune passion pour la mon- tagne pouvaient, au contraire, les rapprocher !

C'est ainsi que les études scientifiques et techniques sur la neige et la glace représentaient le lieu de rencontre neutre tout désigné entre les ingénieurs des deux disciplines.

\section{Un cadre idéal : la SHF}

A cette époque, la Société Hydrotechnique de France, dont la revue s'appelait précisément "La Houille Blanche " était déjà le centre de rencontre naturel des hydroélectriciens d'EDF.

Introduire, par le biais de l'eau solide, glace ou neige, les forestiers dans la SHF fut la proposition que M. Messines eut l'idée de génie de faire à M. BARILLON, alors Président du Comité technique de la Société. Il en reçut un accueil enthousiaste et le Président BARILLON devait être, durant de longues années, un membre des plus assidus tant des réunions que des tournées de notre section.

\section{La réunion fondatrice du 21 décembre 1948}

C'est le 21 décembre 1948 qu'eut lieu la réunion constitutive de ce qui ne fut, au départ qu'une "sous-section " de la SHF mais qui devait, très vite, vu son succès. devenir une section à part entière.

On donne en annexe (1) la liste des 22 participants à cette première réunion : forestiers et hydroélectriciens y sont à égalité, et nettement majoritaires ( 7 et 7) mais on y trouve aussi 4 membres des expéditions polaires françaises (dont Paul Emile VICTOR) ainsi que deux géographes (MM. ONDE et de MARTONNE) et un ingénieur des Ponts et Chaussées. Cette première réunion établira une « liste de membres" (voir annexe II) ayant fait connaître leur adhésion.

Beaucoup plus complète, cette liste des adhérents de la première heure comprend nombre de personnalités scientifiques, en particulier grenobloises. Mais elle énumère également un nombre important de membres correspondants étrangers éminents, suisses, italiens, suédois, américains, anglais, auxquels se joindront très vite des amis fidèles autrichiens, espagnols, yougoslaves, chiliens! 


\section{Un programme ambitieux}

Le programme d'activités annoncé lors de cette première réunion est ambitieux et recouvre déjà un certain nombre des domaines qui seront abordés par nos membres au cours des décennies suivantes :

- identification et nomenclature des glaciers français,

- observations glaciologiques par relevés topographiques et études de mouvement des langues, mais aussi par la méthode des bilans glaciaires dite d'Ahlman (le célèbre glaciologue suédois était correspondant de notre jeune section) qui venait d'être initiée à Sarennes par M. CHERREY.

- études des radiations solaires sur la glace et la neige,

- étude des débits des torrents glaciaires (qui intéressaient prodigieusement EDF),

- études de l'érosion glaciaire,

- études sur la dynamique des glaciers. MM. BARILLON et RÉMÉNIÉRAS évoquaient déjà la possibilité d'une étude sur modèle réduit avec une matière plastique à déterminer !

En matière de mesures:

- Après critique et discussions des instruments traditionnels de mesure des précipitations neigeuses (tables à neige et totalisateurs Mougin) EDF expose ses projets qui contiennent en germe tout ce qui sera plus tard mis au point par sa Division Technique Générale. notamment au Col de Porte.

- Une revue comparative est passée des différents types de sondes thermiques ou mécaniques alors imaginées pour les explorations sous-et intra-glaciaires.

- Enfin M. Sulzlee, un forestier pyrénéen. donne des précisions sur les données collectées par les services forestiers concernant les avalanches comme sur ses propres études conduites à Barèges sur cette question. On voit que, dès son origine, la section de glaciologie était ouverte à un très large éventail de domaines, mais, conformément à la vocation propre de la SHF, société d'ingénieurs, elle a toujours insisté sur les applications pratiques des recherches scientifiques qu'elle se donnait pour rôle de susciter ou d'encourager.

\section{Des réunions annuelles den- ses, variées et appréciées}

Les réunions annuelles de février ou mars à Paris eurent vite un succès appréciable et la salle du 199 rue de Grenelle était souvent trop petite ! Leurs programmes ne différaient guère de ce qu'ils sont encore aujourd'hui permettant à chaque spécialiste de se tenir au courant des progrès et des préoccupations de ses homologues de disciplines voisines :

- physique et mécanique de la glace : dès la création, tant attendue, d'une équipe de glaciologie fondamentale à Grenoble avec le Professeur LLIBOUTRY vers 1957, elle fut évidemment très sollicitée pour de nombreuses communications de ses chercheurs,

- glaciologie alpine : variations des glaciers tempérés avec les communications de nos amis suisses et italiens et des forestiers français (avec le traditionnel compte rendu sur les mesures de Sarennes),

- glaciologie polaire : deux ou trois communications sur les plus récents acquis des expéditions polaires françaises : la section eut la primeur des remarquables résultats de l'équipe LORIUS sur l'histoire des climats à partir de carottes de glace antarctique,

- aménagements hydroélectriques : plusieurs communications d'hydroélectriciens suisses, italiens ou français sur des problèmes d'équipement, de mesures de ressources en neige et de prévision d'apport avec les résultats acquis au Col de Porte,

— avalanches : nombreuses communications sur les progrès réalisés par la météo dans le domaine de la prévision, comptes rendus d'accident, prévention et défense par la division nivologie, l'ANENA, etc...,

- morphologie des zones proglaciaires, glaciers rocheux, moraines, sols polygonaux, érosion glaciaire, torrents glaciaires, végétation et études palynologiques sur les marches proglaciaires, etc... étaient toujours l'objet d'une ou plusieurs interventions de différents chercheurs, notamment de géographes,

- parfois une communication "vedette " telle que celle de Michel SirFrE, à peine sorti de sa réclusion spéléologique volontaire pour étudier son propre rythme nycthéméral !

Si on y ajoute les comptes rendus de la dernière tournée, la préparation de la prochaine et les « analyses bibliographiques " (j'ai un souvenir cuisant des analyses de tous les articles du " Journal of Glaciology " qu'exigeait M. MEssines !), on voit que la journée et demie que duraient ces réunions étaient souvent plus que denses, mais toujours suivies avec passion.

\section{Des tournées riches et convi- viables}

Les tournées glaciologiques eurent un succès encore plus vif si c'est possible ! J'ai essayé, en annexe 3 , d'en reconstituer la liste depuis l'origine ; rares sont les massifs glaciaires européens qui n'ont pas été visités au moins une fois par la section. Le programme type comprenait la visite d'un ou plusieurs glaciers, celle d'un équipement hydroélectrique, d'un site torrentiel ou d'avalanche et très souvent d'un site présentant un intérêt scientifique d'ordre plus général, laboratoire, parc national, etc... Toujours, il y avait, au moins pour le groupe $A$, une nuit en refuge où la bonne humeur entretenue par M. MESSINES et quelques autres faisait merveille. Nombres d'amitiés durables se sont forgées entre des personnes qui ne seraient jamais rencontrées sans la section de glaciologie. Beaucoup d'ingénieurs ou de chercheurs, trop souvent rivés à leurs bureaux le reste de l'année, n'auraient pour rien au monde, renoncé à cette « coupure " annuelle, si dure qu'elle fût sur le plan physique et tous disent $y$ avoir appris plus que dans les ouvrages spécialisés.

Indiscutablement, l'objectif initial de ren- contre et de création de liens personnels entre des ingénieurs de formation et de responsabilités diverses a été magnifiquement atteint !

\section{Les faits saillants de $\mathbf{4 5}$ ans de la vie de la section}

La suite de l'histoire de la section montre la richesse des réalisations qu'elle a permises ou suscitées.

1955 Le CEDONIGLA et le laboratoire du Col de Porte

C'est à la suite des contacts établis à la section de glaciologie de la SHF que fut créée, en 1955, le "CEDONIGLA " (Centre d'Etudes et de Documentation Nivo-Glaciologique) qui rassemblait :

- La $7^{\mathrm{c}}$ section de recherches forestières (lointain « ancêtre " de l'actuelle division nivologie du CEMAGREF). Dépendant, à l'époque, de l'Administration des Eaux et Forêts, cette section, animée par Louis GARAVEL, a en charge le problème des risques d'avalanches et cherche à lancer des études sur le manteau neigeux. Elle peut disposer d'un terrain facile d'accès et bien enneigé dans un coin de forêt domaniale au Col de Porte, mais n'a pas les moyens, ni en hommes ni en matériel, de créer le laboratoire souhaité.

- La Division Technique générale d'EDF qui cherche à acquérir plus de connaissances sur le dépôt et la fusion de la neige au sol et veut mettre au point un instrument moderne de mesure (le futur télénivomètre) dispose de matériel mais manque de chercheurs et de terrain.

- La Météorologie Nationale tout à fait consciente de la nécessité prochaine d'accéder à la prévision du risque d'avalanches dispose de personnel (M. PoGGi, M. PLAS) mais de peu de moyens en matériel et n'a pas de terrain d'expérience.

Le pragmatisme efficace de Louis GARAVEL eut vite fait de rassembler les atouts de chacun et de monter une coopération pratique performante, totalement inimaginable et d'ailleurs ignorée des bureaux parisiens. entre deux administrations et un établissement public qui dépendaient de trois ministres différents ! Le CEDONIGLA a d'abord rassemblé et diffusé dans son bulletin des données appréciées sur la neige et les avalanches dans les Alpes, puis très vite a créé le Laboratoire du Col de Porte qui fut précieux pour EDF et qui demeure essentiel pour l'actuel « Centre de la neige $»$ de la Météo.

1957/58 Le Laboratoire de Glaciologie

Retour du Chili, Louis Lliboutry, ancien collaborateur de Louis NEEL, crée la chaire de glaciologie qui manquait tant à Grenoble 
et à la France. Il est, avec son équipe, un assidu de nos réunions et de nos tournées, où ses collaborateurs apportent aux ingénieurs de la SHF des bases scientifiques solides et une caution inappréciable au plan international vis-à-vis des instances scientifiques en géophysique (comité “ neiges et glaces » de l'AIHS, UGGI, etc...). Dès cette époque. Grenoble était devenu le pôle de toutes les études sur la neige et la glace en France.

\section{L'ONF et l'arrêt des observa- tions glaciologiques}

La création de l'Office National des Forêts devait entrainer la répartition des agents de l'ancienne Administration des Eaux et Forêts en deux groupes: les uns chargés d'appliquer la législation forestière aux forêts privées rejoignirent les jeunes DDA et n'avaient ni le temps ni les moyens de procéder aux observations glaciologiques traditionnelles poursuivies par les forestiers depuis le début du siècle. Les autres furent rassemblés dans un Etablissement Public à caractère industriel et commercial, I'Office National des Forêts, exclusivement chargé de la gestion des forêts publiques, domaniales et communales, et financé par cette seule gestion ! II était difficile d'assimiler les glaciers des Alpes et des Pyrénées à des forêts de rapport, et le combat qu' un certain nombre d'entre nous menèrent pour que l'ONF poursuive ces mesures avait, il faut le reconnaître, bien peu de chances d'aboutir ! II fallut plusieurs années et un sérieux risque d'interruption durable, qui aurait été catastrophique, pour qu'une solution de remplacement fut enfin trouvée à la charge du Ministère de l'Agriculture par la Division Nivologie qui procéda au rattachement à la triangulation générale de repères autour des principaux glaciers, permettant des relevés précis par photogrammétrie aérienne périodique.

1968 Les Jeux Olympiques de Grenoble et le Centre d'études de la Neige

Depuis l'époque du CEDONIGLA, la Météorologie Nationale n'avait cessé d'accroître sa participation aux travaux du Col de Porte et de manifester son intérêt croissant pour les techniques d'examen du manteau neigeux et pour les prévisions d'avalanches qui en dépendaient. Répondant à une commande précise du "Cojo \#, le Centre d'étude de la neige dut accroître rapidement ses moyens au point de prendre directement à partir des années 1970 la plus grande part de la responsabilité du laboratoire du Col de Porte.
1970/71 La Commission dite «de Val d'Isère et la création de I'ANENA "

La commission interministérielle d'enquête créée par le Gouvernement à la suite de l'avalanche tragique de Val d'Isère $(10 / 2 / 1970)$ fut chargée de rechercher qui, en France, s'occupait de neige, de glace et d'avalanches. Elle ne pouvait trouver ces compétences que parmi les membres de la section de Glaciologie de la SHF, en particulier au CEDONIGLA et au laboratoire de Glaciologie. La création en octobre 1971 de I'Association Nationale pour I'Etude de la Neige et des Avalanches (ANENA) fut la conséquence directe d'une recommandation pressante de la Commission. Dès sa naissance, les responsables de I'ANENA, parmi lesquels le laboratoire des applications spéciales de la physique du CENG, dont le chef était un ancien des expéditions polaires françaises, furent des membres actifs et assidus de notre section qui est régulièrement et abondamment tenue au courant des activités de la jeune association.

\section{Les relations avec l'IGS}

De longue date, la section de glaciologie de la SHF entretenait des relations étroites avec la Société britannique de Glaciologie qui, de royale, était devenue " internationale " dès qu'elle eut constaté que la majorité de ses membres étaient américains. Mais I'IGS (International Glaciological Society), qui publiait la seule revue scientifique mondiale de la spécialité (le " Journal of Glaciology ") ainsi que le bulletin "Ice ", avait toujours son siège à Cambridge. «Ice " rendait d'ailleurs compte, assez régulièrement de nos activités.

Cependant, un certain nombre d'universitaires français avaient manifesté le souhait de former une section de l'IGS, notamment pour avoir plus facilement accès aux colonnes du " Journal of Glaciology ". Une revendication bien légitime. Mais la constitution de la section française (ou plutôt francophone) dite $\propto$ des Alpes Occidentales " de I'IGS entraînait la nécessité d'une réunion annuelle avec éventuellement des communications et une tournée glaciologique également annuelle.

Le public intéressé par cette nouvelle section était manifestement le même que celui de notre section de la SHF. Une concurrence ou une rivalité eussent été désastreuses et tout à fait contraires à notre objectif initial !

Un « modus vivendi » fut trouvé consistant à établir en commun les programmes des réunions et des tournées pour qu'elles se succèdent sans recouvrement ni hiatus dans le temps comme dans l'espace et que chacun puisse commodément assister aux deux manifestations s'il le désire.
Les années 70 et 80 : l'alternance Paris-Grenoble, le recentrage à Grenoble

M. Messines dirigeait déjà de Grenoble les services de la RTM (Restauration des Terrains en Montagne).

L'Institut de Géographie Alpine, la Division Technique Générale d'EDF, la $7^{\mathrm{e}}$ section de recherches forestières et la Division Nivologie du CEMAGREF qui lui a succédé, puis le CEDONIGLA, le Laboratoire de Glaciologie, le centre d'étude de la neige, enfin l'ANENA et le laboratoire des Applications spéciales de la physique du CENG, bref l'immense majorité des membres actifs ou potentiels de la section de glaciologie de la SHF étaient et sont toujours basés à Grenoble. Ils en font la capitale française des études sur la glace et la neige et il était naturel, en cette époque de décentralisation, que le centre de gravité de la section de glaciologie fût attiré par Grenoble - d'autant que la salle que le CEMAGREF peut mettre à notre disposition ici est sans commune mesure du point de vue de ses dimensions avec les étroits locaux de la rue de Grenelle! Les responsables parisiens de la SHF ont bien voulu. malgré le surcroît de travail qui en résultait pour eux, accepter d'abord l'alternance de nos réunions en salle, une année à Paris. l'année suivante à Grenoble, puis, désormais, que toutes les réunions se tiennent dans la capitale des Alpes. Qu'ils en soient vivement remerciés.

La section de glaciologie de la SHF a 45 ans. Le bref rappel historique qui précède est très loin d'épuiser tout ce que ses membres ont retiré et retirent encore de ce cadre qu'elle offre à leurs activités : lieu de rencontre, plus que jamais, lieu de sympathie complice née des tournées et lieu d'acquisition de connaissances et de relations fécondes.

Pour ce qui est des programmes, les projets exprimés lors de la réunion inaugurale du 21 décembre 1948 ont été très largement exécutés : le monumental ouvrage de Robert VIVIan publié en 1975 sur les glaciers des Alpes Occidentales dépasse, de loin, le souci d'identification et de nomenclature des glaciers, nos connaissances et les mesures faites de nos jours en France sur la neige, la mécanique des glaciers, l'hydrologie nivale et glaciaire, la prévision et la défense contre les avalanches sont très audelà de ce que souhaitaient atteindre les participants de notre première réunion, le 21 décembre 1948 !

C'est naturel, mais, comme toujours en matière de progrès scientifiques, le nombre de questions nouvelles qui se posent sont à la mesure de ces progrès eux-mêmes et vous $n$ 'aurez pas trop des prochains 45 ans pour les résoudre! 


\section{L'EAU, L'HOMME et LA NATURE}

\section{Les Sciences hydrotechniques au service du développement durable}

\section{Appel à communications}

\section{Rapporteurs Généraux}

\section{F. VILLEY-DESMESERETS,} Ingénieur général à la Mission d'inspection spécialisée du Ministère de l'Environnement, (M.I.S.E.), Paris.

\section{J.L. BESEME,}

Ingénieur en chef du GREF, Directeur de l'Agence de l'eau Loire-Bretagne, Orléans.

\section{L.A. ROMAÑA,}

Directeur du Laboratoire "chimie des contaminants et modélisation" à la Direction de l'Environnement de l'IFREMER, La Seyne sur Mer.
Les 24èmes journées de I'hydraulique se proposent de poursuivre, particulièrement dans le domaine des eaux continentales. le travail de réflexion lancé en 1992 lors des 22 èmes journées de l'hydraulique par l'examen panoramique de I'Avenir de I'Eau. Elles s'inscrivent dans le courant essentiel de réflexions sur les rapports entre la nature, avec ses propres mécanismes d'évolution, et l'homme, une des dernières et des plus perfectionnées des créations de la nature, mais, qui, de ce fait même, peut avoir sur elle une action importante, favorable ou défavorable. Les journées reprennent, avec la volonté de les réactualiser par les apports de réflexion sociologique et scientifique qui se sont depuis largement développés, les sujets abordés de longue date par la S.H.F. tels que "I'influence des activités de I'homme sur le cycle hydrométéorologique" (1974), "I'hydraulique et la maîtrise du littoral" (1984). "l'impact des activités humaines sur les eaux continentales" (1986), "I'aménagement doux des fleuves et rivières" (1991).

Pour relier l'étude des eaux continentales à leur genèse et à leur devenir, le corps principal de ces 24èmes journées que constitue le thème 2 (Les Chemins de l'Eau) est introduit par un thème sur le cycle de l'eau (thème 1).

* Le présent appel à communications porte principalement sur le thème 2 , qui intéresse l'eau, des précipitations jusqu'aux effets en mer, hydrographiques, mécaniques, écologiques. $\mathrm{Ce}$ thème a été divisé en 3 questions :

. le bassin, qui reçoit les précipitations, et où I'action de I'homme, même lorsqu'elle n'a pas un but directement hydraulique, a des conséquences très importantes sur l'eau, par l'intermédiaire de tout le chevelu des écoulement superficiels et phréatiques,

. les rives, les rivières où se cristalliser les principales actions des riverains st les cours d'eau,

- I'interface du réseau des cours d'ea avec la mer qui intègre, mécaniquemer et écologiquement, toute I'histoir hydrologique de l'amont.

$*$ le thème 1 doit être surtout trait par une discussion organisée autour $d$ deux exposés fondamentaux présenté par deux conférenciers invités.

Cependant, nous avons souhaité appuyt visuellement ce thème par un larg panorama de posters graphiques 0 audiovisuels portant sur tous le domaines des sciences et techniques $d$ l'eau, spécialement dans les domaines qi ne sont pas développés dans le thème: Des propositions en ce sens sor demandées: elles seront examinées e même temps et au même titre que le propositions de communications.

* Le thème 3 ne fait pas I'objı d'appel à communications : une TABL RONDE de spécialistes de formations , de responsabilités diverses, qui aurot pris part, directement ou pi I'intermédiaire des rapporteurs générau: aux débats des thèmes 1 et 2 concluı dans un sens prospectif afin d'aider le scientifiques, aménageurs et décideurs unir leurs efforts pour une actio harmonieuse de développement durable.

P. CAZENAVI Président du Comité Scientifique , Technique de SOCIETE HYDROTECHNIQUE DE FRANC 
Les sciences hydrotechniques au service du développement durable

\section{THEME 1 : LE CYCLE DE L'EAU ET SES UTILISATIONS}

Le cycle de l'eau, mers et continents: . Que sait-on de nouveau du cycle de l'eau ?

. Quelles sont les actions de l'homme sur ce cycle?

- 2 exposés introductifs présentés par des conférenciers invités

- Posters fixes ou animés avec brève séance de présentation.

- Discussions en ateliers.

\section{THEME 2 : LES CHEMINS DE L'EAU}

(Communications particulières)

\section{QUESTION 1. - Les bassins versants}

\section{Rapporteur général : F. VILLEY-DESMESERETS}

- les bassins dans la politique de l'eau : les agences de l'eau et les SDAGE.

les bassins en hydrologie continentale ; relations entre les écoulements et les bassins versants

- effets des aménagements urbains et ruraux, des exploitations industrielles, agricoles, touristiques...

. bassins versants naturels ou entretenus,

. bassins versants aménagés ou saccagés.

\section{QUESTION 2. - Rives, rivières, riverains} et écosystèmes aquatiques

\section{Rapporteur général : J.L. BESEME}

rivières naturelles ou entretenues, rivières aménagées ou saccagées.

- utilisation des rivières et respect des écosystèmes aquatiques :

aménagement intégré et développement durable agriculture, alimentation en eau potable, hydroélectricité, navigation, phénomènes de désertification, protection contre les inondations, rejets urbains et industriels, soutien des étiages, tourisme ...

. les zones humides : de la malédiction des marais à la reconnaissance de l'importance écologique des zones humides.

\section{QUESTION 3. - Deltas, estuaires et littoral}

Rapporteur général : L.A. ROMAÑNA

. influence de l'entretien et de l'usage des bassins versants continentaux sur l'évolution des embouchures et du littoral et sur la qualité des eaux marines :

transports solides, pollutions,

évolution des écosystèmes du littoral, etc... . recul des côtes et protection du littoral.

\section{THEME 3 : UTILISATION DE L'EAU ET SAUVEGARDE DE LA NATURE - PROSPECTIVE}

Prospective pour le 21 ème siècle (exposé par un conférencier invité)

TABLE RONDE

L'appel porte sur les propositions de posters (graphiques ou vidéos) du thème $\mathbf{1}$ et sur les propositions de communications du thème 2 . Chaque question du thème 2 sera traitée en deux parties : la première réservée au rapport général, la deuxième consacrée à un exposé des communications suivi de discussion.

\section{1.-Propositions de communications et posters}

Les résumés de communications, ou les descriptions de posters, limités à une page dactylographiée, peuvent être adressés, dès maintenant, à la SOCIETE HYDROTECHNIQUE DE FRANCE, 199 rue de Grenelle, 75007 PARIS, qui transmettra aux Rapporteurs généraux intéressés.

Ils doivent comporter : . Nom, prénom, fonction, titre de l'auteur principal et des co-auteurs

. Adresse, numéro de téléphone et de fax de l'auteur principal

. Titre du poster ou de la communication proposée

Une première sélection sera faite par les rapporteurs généraux en mai 1995, les textes étant toutefois pris en considération jusqu'à la date limite du

\section{SEPTEMBRE 1995}

Les décisions relatives aux différentes propositions seront notifiées aux auteurs principaux au plus tard le 20 décembre 1995 ainsi que les dispositions pour la présentation matérielle.

\section{2.- Présentation des communications}

Les textes des communications retenues seront édités par la SOCIETE HYDROTECHNIQUE DE FRANCE en un recueil qui sera remis à tous les participants ayant acquitté les droits d'inscription correspondants. Les originaux comprenant environ 8 pages (y compris les illustrations) seront à remettre à la SOCIETE HYDROTECHNIQUE DE FRANCE, au plus tard le 31 MARS 1996. 